Journal of

Cancer Research and Therapeutic Oncology

\title{
Improving Detection of Somatic Mutations
}

Thurai Moorthy"

President/CSO, MultiGEN Diagnostics Inc, 1100 Revolution Mill Drive, Greensboro, NC 27405, USA

${ }^{\star}$ Corresponding author: Thurai Moorthy, President/CSO, MultiGEN Diagnostics Inc, 1100 Revolution Mill Drive, Greensboro, NC 27405, USA;Tel: (858) 356 7492; E-mail: Moorthy@multigen-diagnostics.com

Received Date: December 06, 2018; Accepted Date: January 28, 2019; Published Date: January 30, 2019

Citation: Thurai Moorthy (2019) Improving Detection of Somatic Mutations. J Cancer Res Therap Oncol 7: 1-2.

Interest in this topic originated from studies on the detection of Braf p. V600E/K mutations using Allele Specific Multiplex Sequencing (ASMS) technology, which was compared to detection of Braf p. V600E by either SNapShot or Ion Torrent. Although there was no discordance among the positives, there was significant discordance among the negatives, indicative of potential false negatives by the latter two methods [1]. A similar pattern was observed when ASMS Braf p. V600E/K mutations was compared to an FDA approved Braf Test (ThxID) (unpublished data). Is there any clinical significance to such discordant results? If so, can testing protocols of somatic mutations be improved to accommodate for potential discrepancies among the test methods so that the results generated could be graded for meaningful clinical interpretation? There are at least four areas that could be affected by such discordant results;

a. Treatment. Some somatic mutations are actionable driver mutations and are considered 'pathogenic' factors [2]. Hence, their detection is critical for patient care. For example, treatment with the kinase inhibitor vermurafenib is based on detecting Braf p. V600E/K mutations in late stage melanoma. Failure to detect these mutations could deprive populations of patients from receiving intended targeted chemotherapy.

b. Monitoring. Use of liquid biopsy is gaining momentum as a method to monitor regression and/or monitor malignancy. For example, EGFR T790M is detected from plasma samples to monitor recurrence of non-small cell lung cancer [3]. Hence, accurate detection of somatic mutations in plasma samples is crucial for patient management, and a false negative could mislead treatment options.

c. Early detection. Presently, somatic mutations are detected in stage IV cancers; however, there are clinical trials to detect them at earlier stages (Eg. stage IIIb, IIIa). Compared to stage IV cancers, stage IIIb/IIIa may have less tumor burden, and hence may be missed by testing protocols that were designed for stage IV cancer. Extending the same argument to population screening, there are somatic mutation-based FDA approved molecular tests to screen for colorectal cancer [4]. Since samples from population screening are pre- cancerous and non-symptomatic, they could carry far lesser numbers of somatic mutant alleles than that of stage IV cancers. Hence, using the test protocol designed for detection in stage IV cancer could lead to false negatives.

d. Clinical trials. Clinical trial protocols for targeted chemotherapy require detection of corresponding somatic mutations to allocate the study patients into respective groups. A potential false negative could lead to grouping patients with somatic mutations with patients who are supposed to have only wild type. A recent publication highlights oversight in clinical trials where the evaluation of detection methods of specific mutations is not well defined, hence the results and conclusion of the trial may stand for re-scrutiny [5].

e. Method comparison. Methods for detection of somatic mutations associated with specific targeted chemotherapy receive their seal of FDA approval through their participation in drug trials. This in turn is followed by development of a number of laboratory developed tests (LDTs) claiming equivalence to FDA approved tests. In doing so, there are several publications comparing different molecular methods for detecting somatic mutations [6,7]. In order to make such comparison meaningful, they have to be based on a fixed amount of DNA input using FFPE samples having specific tumor content or a formula to integrate various amounts of DNA and the tumor content used. 
Present molecular tests use a range of platforms; Realtime PCR, Next generation sequencing, Sanger sequencing, pyrosequencing, primer extension, etc. All these tests use various amounts (10 ng to $1000 \mathrm{ng}$ ) of extracted DNA from FFPE samples. Further, tumor content of FFPE samples varies from $1 \%$ to $100 \%$. Failing to integrate the two determinants (number of copies of mutant allele and tumor content), tumor content was adopted as a sole parameter to measure efficacy of detection, overshadowing the limit of detection. Although such an approach conforms to CLIA regulations, it fails to determine relative efficacy of each method and the clinical validity of the results generated. Further, the freedom to use arbitrary combinations of the amount of DNA and tumor content has led to non-uniformity of the testing process. Although some molecular tests were validated through drug trials for specific diagnosis and treatment, by default those assays invariably become the accepted method of choice for detecting somatic mutations in all cancer types. Unfortunately, the absence of proper vetting processes has created a favorable environment for molecular tests that were primarily developed for infectious diseases and/or research to find their way into testing somatic mutations, carrying their limitations with them. Laboratory investigations are adopted based on the status of the analyte in respective sample matrices, and not on the limitations of the methods used. However, all these methods are here to stay, and hence there has to be a way to accommodate them while at the same time provide a meaningful interpretation of, the results they generate. Irrespective of whether the molecular test is an FDA approved test or an LDT, presently there is no uniformity among testing protocols or the measure of efficacy of testing by various methods used for detecting somatic mutations.

To overcome these challenges, a new criterion, Detection Index (DI), was formulated [8]. Detection Index is based on the two basic determinants of detection, the limit of detection of the mutation, and the ratio of the copies of the mutant allele to that of the wild type. Hence, DI achieves several purposes; firstly, one could objectively define a level of detection to evaluate the efficacy of detection of somatic mutations beyond stage IV cancers, including early stages, liquid biopsy and population screening independently. Secondly, DI allows all molecular tests to be inclusive to achieve desired DI by adjusting the two basic factors, the amount of DNA and/or tumor content. Thirdly, in the future, quantifying somatic mutations in clinical trials will be inevitable. Hence, DI will enable drug developers to make a claim within a specific range of DI for a specific drug, and allow clinical laboratories to design test protocols to match the DI that was claimed for the drug. Finally, DI will serve as a meaningful scientific terminology among stake holders, including the drug manufacturers, IVD manufacturers, regulatory (FDA) and auditing organizations (CAP), and for CMS-CLIA approval involved in molecular diagnosis. A simple parameter, Detection Index, reflects the efficacy of detecting somatic mutations, and will be very helpful in setting testing standards, as well as for meaningful clinical interpretation.

\section{References}

1. Vinayagamoorthy T, Zhang D, Ye F, Vinayagamoorthy D, Hodkinson R (2017) Can detection of Braf p.V600E mutation be improved? Comparison of allele specific multiplex sequencing to present tests. Journal of Solid Tumors. 7:2.

2. Mehnert JM and Kluger HM (2012) Driver Mutations in Melanoma: Lessons Learned From Bench-to-Bedside Studies. Curr Oncol Rep. 14: 449-457.

3. Mellert H, Foreman T, Jackson L, et al. (2017) Development and Clinical Utility of a Blood- Based Test Service for the Rapid Identification of Actionable Mutations in Non-Small Cell Lung Carcinoma. 19: 3 404-416.

4. Yoruker EE, Holdenrieder S, Gezer U (2016) Blood-based biomarkers for diagnosis, prognosisand treatment of colorectal cancer. Clinica Chimica Acta. 455: 1 26-32.

5. Moorthy T (2018) Targeted therapy: a potential oversight in trial protocol. The Lancet Oncology. 19: 39.

6. Prieto-Potin I, Montagut C, Bellosillo B, et al. (2018) Multicenter Evaluation of the Idylla NRAS-BRAF Mutation Test in Metastatic Colorectal Cancer. J Mol Diagn. 20 :664-676.

7. Kevin Q, Qiulu P, Xi Z, et al. (2013) Detection of BRAF V600 Mutations in Metastatic Melanoma. Comparison of the Cobas 4800 and Sanger Sequencing Assays. The Journal of Molecular Diagnostics. 15: 790.

8. Moorthy T (2018) An algorithm to evaluate the efficacy of detecting somatic mutations. Journal of Solid Tumors 8: 2 .

\section{Submit your manuscript to a JScholar journal and benefit from: \\ ฯ Convenient online submission \\ ฯ Rigorous peer review \\ - Immediate publication on acceptance \\ ฯ Open access: articles freely available online \\ ब High visibility within the field \\ I Better discount for your subsequent articles Submit your manuscript at http://www.jscholaronline.org/submit-manuscript.php}

